\title{
AJUSTE PSICOLÓGICO Y HABILIDADES PARA LA RESOLUCIÓN DE PROBLEMAS EN CONDENADOS POR HOMICIDIO O TENTATIVA DE HOMICIDIO
}

\author{
PSYCHOLOGICAL ADJUSTMENT AND \\ PROBLEM-SOLVING SKILLS IN CONVICTED \\ BY MURDER OR ATTEMPTED MURDER
}

\author{
MANUEl Vilariño ${ }^{1}$, BARBARA G. AMADO ${ }^{2}$, Elsa FERNANDES ${ }^{2}$ \\ Y RAMÓN ARCE ${ }^{3}$
}

Cómo referenciar este artículo/How to reference this article:

Vilariño, M., Amado, B. G., Fernandes, E. y Arce, R. (2017). Ajuste psicológico y habilidades para la resolución de problemas en condenados por homicidio o tentativa de homicidio [Psychological Adjustment and Problem Solving Skills in Convicted by Murder or Attempted Murder]. Acción Psicológica, 14(2), 129-146. https://doi.org/10.5944/ap.14.2.20792

\begin{abstract}
Resumen
Con el objetivo de conocer el ajuste psicológico (sintomatología y diagnóstico clínico) y las destrezas para la resolución de problemas (afrontamiento) en una población de homicidas, se ha llevado a cabo un estudio en el que se contrastaron ambos factores entre una muestra de condenados por homicidio o tentativa de homicidio, con la población normativa. Participaron en el estudio 27 hombres internos en el Centro Penitenciario de Oporto
\end{abstract}

(Portugal), con una edad promedio de 38.63 años. Para la medida del ajuste psicológico se acudió al SCL-90-R, mientras que el afrontamiento fue evaluado mediante el CRI-A. Los resultados revelaron que los reclusos mostraban una probabilidad superior de ser clasificados como casos clínicos en las dimensiones clínicas del SCL90-R, a excepción de Hostilidad, así como puntuaciones significativamente más elevadas en las dimensiones clínicas a excepción de Hostilidad y Ansiedad Fóbica, y en los tres índices globales (i.e., GSI, PST, PSDI). Además, la muestra de condenados se caracterizaba por acudir a estrategias de afrontamiento evitativas, que son recono-

Correspondencia: Bárbara G. Amado, Unidade de Psicoloxía Forense. Universidade de Santiago de Compostela, España. Email: barbara.gonzalez.amado@usc.es

ORCID: Manuel Vilariño ( https://orcid.org/0000-0001-8212-3319) Barbara G. Amado (http://orcid.org/0000-00019262-1469)Elsa Fernandes (http://orcid.org/0000-0001-9612-7572) y Ramón Arce (http://orcid.org/0000-0002-56223022).

${ }^{1}$ Departamento de Psicología y Sociología, Universidad de Zaragoza, España.

${ }^{2}$ Unidade de Psicoloxía Forense, Universidade de Santiago de Compostela, España.

${ }^{3}$ Facultade de Psicoloxía, Universidade de Santiago de Compostela, España.

Recibido: 09 agosto de 2017.

Aceptado: 29 de octubre de 2017. 
cidas como formas desadaptativas o improductivas. Asimismo, del estudio de las características delictivas se verificó que la mayoría de los acusados guardaban algún tipo de relación previa con la víctima, y que la muestra homicida se caracterizaba por versatilidad delictiva y tendencia a la reincidencia. Se discuten las implicaciones de estos resultados para la práctica profesional en el contexto penitenciario.

Palabras clave: Ajuste psicológico; Sintomatología clínica; Habilidades de resolución de problemas; Afrontamiento; Homicidio; Reclusos.

\begin{abstract}
With the aim of knowing about psychological adjustment (clinical symptomatology and diagnosis) and problemsolving skills (coping) in a homicide prison inmate population, a study to contrast sentenced murderers or attempted murderers with the normative population was conducted. Participated in the research, 27 male prison inmates at the Prison of Oporto (Portugal) with an age average of 38.63. Psychological adjustment and coping strategies were measured with the SCL-90-R checklist and the CRI-A questionnaire, respectively. The results exhibited that prison inmates showed a higher probability of being classified as clinical cases in most of the SCL90-R dimensions (except in Hostility), as well as significantly higher scores in almost all of them (with the exception of Hostility and Phobic Anxiety), an in the global indexes (i.e., GSI, PST, PSDI). Furthermore, prison inmates were characterized by using avoidance coping strategies, which are considered such as maladaptive or unproductive. Likewise, the results from the criminological characteristics of the inmates have revealed that homicides had a previous relationship with the victim, criminal versatility and a recidivism trend. Implications of the results for professional practice in penitentiary setting are discussed.
\end{abstract}

Keywords: Psychological adjustment; Clinical symptoms; Problem solving skills; Coping; Murder; Prison inmates.

\section{Introducción}

El homicidio atenta contra el bien jurídico por antonomasia que protege el Derecho Penal, la vida humana. En consecuencia, es reprobado socialmente, a la vez que suele llevar asignadas las condenas más altas. Desde un punto de vista epidemiológico, aunque se aprecia un descenso en la incidencia, comparando la tasa media entre los años 2007-2009 y 2010-2012 (Eurostat Statistics Database, 2014), continúa siendo una problemática preocupante por las graves e irreversibles consecuencias que entraña.

Tradicionalmente, para la explicación de la conducta delictiva y antisocial, en general, y del homicidio, en particular, se han desarrollado tres grandes enfoques que difieren en la importancia concedida a la etiología que subyace la conducta desviada: la aproximación biológica, la sociológica, y la psicológica (Botelho y Gonçalves, 2016). Sin embargo, la complejidad de la conducta humana dificulta adoptar una aproximación reduccionista para explicar cualquier acto, sea o no delictivo. De este modo, se han propuesto nuevos modelos y perspectivas que entienden el comportamiento delictivo como el resultado de la interrelación de distintos factores de riesgo y protectores (Arce, Fariña y Vázquez, 2011; Connell, Cook, Aklin, Vanderploeg y Brex, 2011; Farrington y Ttofi, 2012). En este sentido, los factores de riesgo representan aquellas variables que incrementan la probabilidad de la emisión de conductas delictivas, mientras que los factores protectores, contrariamente, la disminuyen (Andrews y Bonta, 2010). Desde estos modelos se contempla, además, un funcionamiento aditivo: cuantos más factores de riesgo reúna una persona, más probabilidades tendrá de delinquir. Por otro lado, las intervenciones preventivas y reeducativas del comportamiento delictivo se dirigen a actuar sobre los denominados factores de riesgo dinámicos (v. gr., ausencia de habilidades sociales) que resultan modificables, frente a los estáticos que no pueden ser alterados (e.g., ser víctima de maltrato infantil).

La enfermedad mental representa uno de los factores de riesgo que se ha asociado al comportamiento delictivo y criminal (Arce, Fariña y Novo, 2014). Múltiples investigaciones abordaron el estudio de la relación entre ambas variables, arrojando resultados contradictorios. Así, 
se han hallado altas tasas de prevalencia de trastornos mentales en reclusos (Fazel y Grann, 2004; Fazel y Seewald, 2012), y un mayor riesgo de conducta homicida en personas con diagnóstico de enfermedad mental (Eronen, Hakola y Tiihonen, 1996; Schanda et al., 2004; Woodward, Nursten, Williamns y Badger, 2000), especialmente con trastornos psicóticos, trastorno antisocial de la personalidad, y abuso de alcohol u otras drogas (RichardDevantoy, Olie y Gourevitch, 2009; Woodward, et al., 2000). Sin embargo, a pesar de la considerable cantidad de estudios que encuentran esta relación, se debe resaltar la ingente dificultad que entraña la predicción de la conducta delictiva, puesto que la mayor parte de las personas con problemas mentales no comenten delitos violentos, y muchos de los delitos violentos no son cometidos por personas con enfermedad mental (Glied y Frank, 2014). En lo que sí se muestra más consistente la literatura, es en que la combinación de ambas variables, enfermedad mental y delincuencia, parece derivar en comportamientos más violentos que cuando aparecen aisladas (Vicens et al., 2011). En este sentido, Silver, Felson y Vaneseltine (2008) encontraron que una historia de tratamiento de enfermedad mental estaba más fuertemente asociada con delincuencia violenta que con otro tipo de delitos. Por tanto, la fusión de los factores delincuencia y enfermedad mental, especialmente cuando ésta es grave, parece redundar en la comisión de delitos graves y violentos como el homicidio.

No obstante, las elevadas tasas de prevalencia de enfermedad mental halladas en población reclusa pueden ser tanto causa como efecto de la propia privación de libertad. La prisionización, el proceso de adaptación al medio penitenciario, genera estrés en la población reclusa. El internamiento implica la privación de libertad, el distanciamiento de la red social y familiar, cambios de ritmo y estilo de vida, y la inmersión en un entorno cultural, social y arquitectónico desconocido y hostil. En suma, el interno se ve obligado a asimilar una nueva cultura y afrontar esta realidad a diferentes niveles: comportamental, cognitivo, emocional y sociocultural. El impacto que la prisionización genera sobre la salud del recluso se ve mediatizado por diversos factores (e.g., la personalidad del preso, el tiempo de condena, el apoyo social percibido), y deriva, generalmente, en la aparición de alteraciones sensoriales y de la imagen personal, pér- dida de autoestima, despersonalización, afectación de la sexualidad, y en sintomatología ansiosa y depresiva (Botelho y Gonçalves, 2016; Ruiz-Hernández, GarcíaJiménez, Llor-Esteban y Godoy-Fernández, 2015; Vilariño y Edmundo, 2013).

Las estrategias de afrontamiento para la resolución de problemas constituyen otro de los factores de riesgo del comportamiento delictivo registrado habitualmente en la literatura (Arce et al., 2014; Moore, Eisler y Franchina, 2000). Estas estrategias se refieren al modo en cómo el individuo aborda los problemas y las situaciones estresantes. En general, de la investigación se extrae que el comportamiento antisocial está relacionado con estrategias de afrontamiento desadaptativas e improductivas, tanto en poblaciones de menores de riesgo y de reforma (Arce, Fariña, Seijo, Novo y Vázquez, 2005; Vilariño, Amado y Alves, 2013), como en poblaciones penitenciarias (Arce et al., 2014).

Por otro lado, emplear estrategias de afrontamiento óptimas dentro del medio penitenciario, podría jugar un papel importante en la salud física y mental del recluso. En esta línea, múltiples trabajos han investigado, en muestras de reclusos, la relación entre afrontamiento y bienestar psicológico o salud mental. Los resultados encontrados han sido, de nuevo, contradictorios (McMurran y Christopher, 2009; Kirchner, Forns y Mohíno, 2007), sin que exista consenso acerca de la forma en cómo afectan las estrategias de afrontamiento al bienestar psicológico del recluso. Por ejemplo, si bien fuera del recinto carcelario se considera que las estrategias centradas en el problema resultan más adaptativas, algunos resultados evidencian que, en el contexto penitenciario y ante determinadas demandas ambientales, las estrategias de tipo pasivo-evitativo se erigen como efectivas y con impacto positivo sobre la salud mental (Van Harreveld, Van der Pligt, Claassen y Van Dijk, 2007). Una posible causa de este resultado parece radicar en que dentro del contexto de prisión se restringen determinadas conductas, lo que aconseja adaptar las estrategias de afrontamiento a la situación específica (Beijersbergen, Dirkzwager, Eichelsheim, Van der Laan y Nieuwbeerta, 2014). 
Ante este estado de la literatura, en el presente trabajo se persigue el objetivo de continuar avanzando en la comprensión de los factores de riesgo que subyacen al comportamiento delictivo, concretamente, al homicidio o tentativa de homicidio. Además, se espera extraer conocimiento útil para la elaboración de programas de intervención preventiva, reeducativa o terapéutica. Por todo ello, se ha llevado a cabo un estudio en el que se contrasta el ajuste psicológico (sintomatología clínica), y las destrezas para la resolución de problemas (estrategias de afrontamiento) de entre una muestra de hombres condenados por homicidio o tentativa de homicidio, con la población normativa. Adicionalmente, para ampliar el conocimiento de la muestra de condenados se han recabado datos de archivo sobre el tipo de homicidio cometido, la relación mantenida con la víctima, y la reincidencia.

\section{Método}

\section{Participantes}

En el estudio participaron 27 hombres que cumplían condena en el 'Estabelecimento Prisional do Porto' (Centro Penitenciario de Oporto, Portugal) por un delito de homicidio o tentativa de homicidio. Sus edades oscilaban entre los 22 y los 65 años $(M=38.63, D T=10.72)$. En relación con el estado civil, el $37 \%$ (10) de los participantes estaban solteros, un $18.5 \%$ (5) formaban parejas de hecho, mientras que casados y divorciados representaban un $22.2 \%$ (6) cada uno de los grupos. En cuanto a la escolaridad, un $63 \%$ (17) poseían estudios de primer ciclo (cuatro años de escolaridad), un $14.8 \%$ (4) carecía de escolaridad; un $7.4 \%$ (2) estudios de tercer ciclo (nueve años escolaridad) y con igual porcentaje los que contaban con estudios secundarios (12 años de escolaridad); un participante $(3.7 \%)$ presentaba estudios de segundo ciclo (seis años de escolaridad), y otro (3.7 \%) poseía estudios superiores.

\section{Diseño y Procedimiento}

Se diseñó un estudio de medida de la sintomatología clínica en una muestra de condenados por homicidio o tentativa de homicidio, contrastando el estado de salud mental de dicha población con la población normativa. Asimismo, se midió la capacidad de afrontamiento mediante el CRI-A en la misma población comparándola, de nuevo, con la población normativa (Moos, 1993). Adicionalmente, se procedió con un estudio de las características delictivas de los condenados para ampliar información relativa al perfil delictivo. Sometida a prueba la sensibilidad del diseño $(1-\beta)$, hallamos, para un tamaño del efecto medio esperado, una sensibilidad de .81, es decir, de ser la media del grupo de homicidas diferente de la de población normativa hay un $81 \%$ de probabilidades de que sea encontrada en este estudio.

La muestra de condenados fue seleccionada de manera accidental entre internos del Centro Penitenciario de Oporto, y su participación fue voluntaria y autorizada. De este modo, la muestra empleada estuvo compuesta por la totalidad de reclusos que cumplían condena por homicidio o tentativa de homicidio en este centro penitenciario, y que dieron consentimiento para participar en la evaluación.

Obtenida la autorización de la dirección del centro, se identificaron los reclusos condenados por homicidio o tentativa de homicidio, con sentencia firme, a partir de los registros informáticos. A continuación, se procedió con la obtención del consentimiento informado de los reclusos para participar en el estudio. Así, se informaba que los datos recabados serían tratados de manera anónima, con meros fines de investigación, y que, en ningún caso, se emplearían en perjuicio del evaluado; que la participación sería voluntaria, y que podrían abandonar el estudio en cualquier momento y sin dar explicaciones. Una vez conseguido el consentimiento, se concertaba una fecha para pasar las pruebas de evaluación y, en su cumplimentación, se reiteraba la necesidad de que fuesen sinceros y que respondiesen de acuerdo con las instrucciones que aparecían redactadas al inicio. Durante la aplicación estaba presente un evaluador para garantizar la correcta cumplimentación, y esclarecer las posibles cuestiones o dudas que pudiesen surgir.

\section{Instrumentos de Medida}

Se aplicó a los participantes la traducción al portugués (back translation) del SCL-90-R [Symptom Check 
List-90-R] de Derogatis (1983) para la evaluación de la sintomatología clínica, y el CRI-A [Coping Responses Inventory Adult Form] de Moos (1993) para la medida de las estrategias de afrontamiento (ver índices de fiabilidad en la Tabla 1). El SCL-90-R fue desarrollado para evaluar patrones de síntomas presentes en el individuo. Consta de 90 ítems en los que la persona informa de sus síntomas psicológicos, psiquiátricos y somáticos. Concretamente, se estructura en torno a nueve dimensiones sintomáticas (i.e., Somatización, Obsesivo-compulsivo, Sensibilidad interpersonal, Depresión, Ansiedad, Hostilidad, Ansiedad fóbica, Ideación paranoide y Psicoticismo), y tres índices globales (i.e., Índice de severidad global, Índice de malestar referido a síntomas positivos, y Total de síntomas positivos).

Por su parte, el CRI-A se compone de dos partes. En la primera, se solicita a la persona evaluada que piense sobre el problema más importante por el que haya pasado en los últimos 12 meses o en su defecto en un problema menor que haya tenido que afrontar y, a continuación, ha de responder a 10 preguntas sobre el mismo para las cuales existen cuatro alternativas de respuesta: nunca, casi nunca, a menudo y siempre. En la segunda parte, se formulan otras 48 preguntas con cuatro alternativas de respuesta: nunca, pocas veces, algunas veces y bastantes veces. El CRI-A evalúa ocho tipos de estrategias de afrontamiento: Análisis Lógico, Reevaluación Positiva, Búsqueda de Orientación y Apoyo, Resolución de Problemas, Evitación Cognitiva, Aceptación o Resignación, Búsqueda de Recompensas Alternativas, y Descarga Emocional.

Tabla 1

Fiabilidad de las dimensiones del SCL-90 y CRI-A

\begin{tabular}{lc}
\hline Variables & Alpha de Cronbach \\
\hline Somatización & .86 \\
Obsesión-Compulsión & .86 \\
Sensibilidad Interpersonal & .86 \\
Depresión & .90 \\
Ansiedad & .85 \\
Hostilidad & .84 \\
Ansiedad Fóbica & .82 \\
Ideación Paranoide & .80 \\
Psicoticismo & .77 \\
\hline
\end{tabular}

\begin{tabular}{lc}
\hline Variables & Alpha de Cronbach \\
\hline Análisis lisis Lógico & .67 \\
Reencuadre positivo & .74 \\
Búsqueda de orientación & .61 \\
Resolución de problemas & .68 \\
Evitación cognitiva & .72 \\
Aceptación o resignación & .64 \\
Búsqueda de actividades & .68 \\
Descarga emocional & .62 \\
\hline
\end{tabular}

\section{Análisis de Datos}

Para el contraste de las puntuaciones obtenidas (sintomatología clínica, y capacidad de afrontamiento) por la muestra de homicidas con los datos de población general normativa (valor de prueba) se acudió a la prueba t para una muestra. Por consiguiente, se ha comparado la muestra de estudio con un valor de prueba dado, estimándose el tamaño del efecto $r$ (Cohen, 1988; Rosenthal, 1994) a partir del estadístico $t$. Para el contraste de una proporción observada con un valor dado o valor de prueba (estudio de casos clínicos) se computó la prueba $Z$, a partir de la cual se estimó el tamaño del efecto $\delta$ de Hedges y Olkin (1985). Adicionalmente, del tamaño del efecto $r$ se obtuvo directamente el incremento en la sintomatología de un grupo respecto del otro (homicidas vs. población general normativa).

\section{Resultados}

\section{Características criminológicas de los condenados por homicidio o tentativa de homicidio}

Con el objetivo de ofrecer una descripción más exhaustiva de los participantes, se procedió con un análisis del historial delictivo del que se extrajo la información

Nota. Índices tomados del manual del SCL-90-R (Derogatis, 1983) y del CRI-A (Moos, 1993). 
Tabla 2

Contraste de la muestra de condenados por homicidio con la media de la población general en las dimensiones sintomáticas del SCL-90. Prueba t para una muestra

\begin{tabular}{lccccc}
\hline Variables & $t$ & $p$ & $M_{\text {Homicidas }}$ & $M_{\mathrm{PG}}$ & $R$ \\
\hline Somatización & 2.98 & .006 & 0.84 & 0.39 & .28 \\
Obsesión-Compulsión & 5.75 & .000 & 1.27 & 0.56 & .49 \\
Sensibilidad Interpersonal & 2.44 & .022 & 0.86 & .43 & .23 \\
Depresión & 4.20 & .000 & 1.24 & 0.59 & .38 \\
Ansiedad & 2.65 & .013 & 0.85 & .25 & .44 \\
Hostilidad & 0.85 & .400 & 0.59 & 0.45 & .08 \\
Ansiedad Fóbica & 1.04 & .307 & 0.27 & 0.19 & .10 \\
Ideación Paranoide & 4.62 & .000 & 1.28 & 0.48 & .41 \\
Psicoticismo & 2.19 & .038 & 0.53 & 0.21 & .21 \\
\hline
\end{tabular}

Nota. $g /(26)$. $M_{\text {Homicidas }}$ Media de condenados por homicidio o tentativa de homicidio; $M_{\mathrm{PG}}=$ valor de prueba o contraste tomado de la Media de la Población General Normativa.

que se refiere a continuación. De los 27 participantes del estudio, el $55.5 \%$ (15) había cometido un delito de homicidio, el $44.4 \%$ (12) homicidio doloso, y el $11.1 \%$ (3) imprudente. El $44.5 \%$ restante cumplía condena por tentativa de homicidio. Con respecto a la relación que mantenían con la víctima, el $59.3 \%$ (16) tenían algún tipo de relación previa (11.1\% pareja, $11.1 \%$ familiar, $37.0 \%$ relación de amistad/vecindad/compañerismo). Por otro lado, el $55.6 \%$ carecía de antecedentes criminales, y de los que contaban con antecedentes sólo uno $(3.7 \%)$ era reincidente en el mismo delito.

\section{Contraste de la sintomatología clínica entre condenados por homicidio o ten- tativa de homicidio y población gene- ral normativa en el SCL-90-R}

En relación con las dimensiones sintomáticas del SCL-90-R, el contraste de las puntuaciones obtenidas por la muestra de condenados con los datos normativos de la muestra de población general normativa recogidos en el SCL-90-R, ha arrojado diferencias significativas en todas las dimensiones, excepto en Hostilidad y Ansiedad Fóbica (ver Tabla 2). Estas diferencias se han concretado sistemáticamente en puntuaciones significativamente superiores en la muestra de condenados. De este modo, los condenados por homicidio o tentativa de homicidio han referido padecer, en contraste con la población general normativa, un $28 \%(r=.28)$ más de signos y síntomas clínicos propios de Somatización (e.g., dolores de cabeza, sensaciones de desmayo o mareo, dolores en el corazón o pecho, nauseas o malestar en el estómago, sentir un nudo en la garganta, etc.); un $49 \%(r=.49)$ más de síntomas de Obsesión-Compulsión (e.g., pensamientos, palabras o ideas no deseadas que no se van de su mente, preocupación acerca del desaseo, el descuido o la desorganización, tener que hacer las cosas muy despacio para estar seguro de que las hace bien, tener que comprobar una y otra vez lo que hace, etc.); un $23 \%(r=.23)$ más de síntomas de Sensibilidad Interpersonal (e.g., ver a la gente siempre de manera negativa, timidez o incomodidad ante el sexo opuesto, la impresión de que otras personas son poco amistosas o que no les gustan, sentirse inferior a los demás, sentirse vergonzoso ante otras personas, etc.); un $38 \%(r=.38)$ más de síntomas propios de Depresión (e.g., pérdida de deseo o placer sexual, sentirse decaído, llorar fácilmente, sentirse solo, sentirse triste, sentirse desesperanzado con respecto al futuro, etc.); un $25 \%(r=.25)$ más de síntomas de Ansiedad (e.g., nerviosismo o agitación interior, temblores, sentirse tenso, ataques de terror o pánico, presentimientos de que va a pasar algo malo, etc.); un $41 \%(r=.41)$ de síntomas de Ideación Paranoide (e.g., impresión de que la mayoría de los problemas son culpa de los demás, sensación de que las otras personas le miran o hablan mal, que los otros no le reconozcan adecuadamente sus méritos, etc.); y un $21 \%$ más de síntomas $(r=.21)$ de Psicoti- 
Tabla 3

Contraste de la muestra de condenados por homicidio con la media de la población general en los índices globales del SCL-90. Prueba "t" para una muestra

\begin{tabular}{lccrcc}
\hline Variables & $t$ & $p$ & $M_{\text {Homicidas }}$ & $M_{\mathrm{PG}}$ & $r$ \\
\hline GSI & 4.02 & .000 & 0.93 & 0.44 & .37 \\
PST & 3.27 & .003 & 35.15 & 22.9 & .30 \\
PSDI & 5.49 & .000 & 2.23 & 1.69 & .47 \\
\hline
\end{tabular}

Nota. $g /(26) . M_{\text {Homicidas }}$ Media de condenados por homicidio o tentativa de homicidio; $M_{\mathrm{PG}}=$ Valor de prueba o contraste tomado de la Media de la Población General Normativa.

cismo (e.g.., la idea de que otra persona pueda controlar sus pensamientos, oír voces que otras personas no oyen, sentirse sólo aunque esté con más gente, sentirse siempre distante sin sensación de intimidad con nadie, etc.).

Por otro lado, como se puede observar en la Tabla 3 , se han detectado diferencias significativas en los tres índices globales del SCL-90-R. Congruentemente con lo registrado en las dimensiones sintomáticas, la muestra de condenados por homicidio o tentativa de homicidio se ha caracterizado por presentar un padecimiento sintomatológico mayor. Concretamente, el GSI informa de un sufrimiento psíquico y psicosomático global significativamente más intenso en la muestra de condenados, que se materializa en un 37\% ( $r=.37)$ más de sintomatología; el PST, en esta misma muestra, evidencia una amplitud y diversidad sintomática significativamente superior que se traduce en un $30 \%(r=.30)$ más de síntomas; mientras que el PSDI pone de manifiesto el padecimiento de una intensidad sintomática media significativamente mayor que se materializada en un $47 \%(r=.47)$ más.

Tabla 4

\section{Estudio de casos clínicos en las dimen- siones SCL-90-R sintomáticas del}

Los estudios de comparación de medias poseen una alta utilidad científica e investigadora, pero resultan insuficientes para una transferencia de conocimiento a la práctica profesional que requiere de diseños de $N=1$. De hecho, esto ha llevado a la American Psychiatric Association (APA, 2002) a recomendar análisis adicionales a la comparación de medias. Consecuentemente, en este trabajo se procedió con el estudio de casos clínicos. Los resultados revelaron en todas las dimensiones sintomáticas del SCL-90-R, excepto en Hostilidad, una prevalencia significativamente superior de casos en la muestra de condenados (ver Tabla 4). Concretamente, en Somatización los homicidas o los condenados por tentativa de homicidio presentan una probabilidad 6.6 veces superior de ser caso clínico en contraste con la población general normativa; en la dimensión de Obsesión-Compulsión la

Prevalencia de casos clínicos en la muestra de condenados en las dimensiones del SCL-90-R

\begin{tabular}{lcrrr}
\hline Variables & Punto de corte & $f(\mathrm{p})$ & \multicolumn{1}{c}{ OR } \\
\hline Somatización & 1.12 & $9(.33)$ & $6.67^{* * *}$ & 6.60 \\
Obsesión-Compulsión & 1.25 & $12(.44)$ & 9.28 & 8.89 \\
Sensibilidad Interpersonal & 1.05 & $10(.37)$ & 7.62 & 7.41 \\
Depresión & 1.37 & $14(.52)$ & $11.19^{* * *}$ & 10.37 \\
Ansiedad & 1.05 & $8(.30)$ & $5.86^{* * *}$ & 5.92 \\
Hostilidad & 1.33 & $3(.11)$ & 1.45 & 2.22 \\
Ansiedad Fóbica & 0.64 & $6(.22)$ & $4.10^{* * *}$ & 4.44 \\
Ideación Paranoide & 1.25 & $11(.41)$ & $8.51^{* * *}$ & 8.15 \\
Psicoticismo & 0.76 & $9(.33)$ & $6.67^{* * *}$ & 6.60 \\
\hline
\end{tabular}

Nota. Como puntos de corte se tomaron las puntuaciones directas que se correspondían con la puntuación T de 65 en cada una de las dimensiones del SCL-90-R, esto es, se tomaron como casos clínicos aquellos con puntuaciones $\geq$ T65 (aproximadamente, $p=.05$ ); ${ }^{*} p<.05 ;{ }^{* *} p<.01$; ${ }^{* *} p<.001$. 
probabilidad asciende a 8.89 veces; en Sensibilidad Interpersonal la probabilidad de ser caso clínico es 7.41 veces mayor; en Depresión es 10.37 veces superior; en Ansiedad de 5.92; en Ansiedad Fóbica la probabilidad de ser caso clínico es 4.44 veces superior; en Ideación Paranoide es 8.15 veces superior; y en Psicoticismo la probabilidad es 6.6 veces mayor. En suma, la probabilidad de ser caso clínico en las dimensiones sintomáticas del SCL-90-R, con la excepción de la dimensión de Hostilidad, es significativamente superior para la muestra de condenados por homicidio o tentativa de homicidio.

\section{Contraste de la capacidad de afronta- miento entre condenados por homici- dio o tentativa de homicidio y po- blación general normativa en el CRI-A}

El contraste en capacidad de afrontamiento (ver Tabla 5) ha evidenciado diferencias en seis de las ocho escalas del CRI-A. La población general normativa sólo presenta una puntuación significativamente superior en la escala Análisis Lógico que se concreta en un $29 \%(r=.29)$ más de empleo de dicha estrategia en comparación con la muestra de condenados. De este modo, los condenados por homicidio se caracterizan por llevar a cabo menos intentos cognitivos de entender y prepararse mentalmente para un estresor y sus consecuencias.
En las restantes escalas en las que se han hallado diferencias, Evitación cognitiva, Aceptación o Resignación, Búsqueda de Actividades Alternativas, y Descarga emocional, era la muestra de condenados la que presentaba puntuaciones significativamente más elevadas. De este modo, la muestra de condenados en contraste con la población general normativa emplea un $34 \%(r=.34)$ más la estrategia de Evitación Cognitiva, que se caracteriza por llevar a cabo intentos cognitivos para evitar pensar de manera realista en el problema. Asimismo, los condenados acuden un $37 \%(r=.37)$ más a las estrategias de Aceptación o Resignación, que se materializa en intentos cognitivos de reaccionar ante el problema aceptándolo. De igual modo, los homicidas recurren un $38 \%$ $(r=.38)$ más a la estrategia de Búsqueda de Actividades Alternativas, que consiste en la realización de actividades sustitutivas y el desarrollo de nuevas formas de satisfacción. Por último, la muestra homicida emplea un $47 \%$ $(\mathrm{r}=.47)$ más la estrategia de Descarga Emocional que implica realizar tentativas de reducir la tensión expresando sentimientos negativos.

\section{Discusión}

Antes de avanzar en la discusión de los resultados hallados en este trabajo, se expondrán algunas limitaciones que se han identificado en el mismo. Primero, se ha empleado una muestra de tamaño reducido, aunque re-

Tabla 5

Contraste de la muestra de condenados por homicidio con la media de la población general de las estrategias de afrontamiento del CRI-A. Valores t para una muestra

\begin{tabular}{lrrrrr}
\hline Variables & \multicolumn{1}{c}{$t$} & $p$ & $M_{\text {Homicidas }}$ & $M_{\text {PG }}$ & $r$ \\
\hline Análisis lógico & -3.14 & .004 & 8.22 & 11.01 & -.29 \\
Reencuadre positivo & -0.95 & .353 & 9.63 & 10.29 & -.09 \\
Búsqueda de orientación & 1.80 & .083 & 10.04 & 8.84 & .17 \\
Resolución de problemas & -0.78 & .443 & 10.26 & 10.82 & -.07 \\
Evitación cognitiva & 3.68 & .001 & 9.15 & 6.66 & .34 \\
Aceptación o resignación & 4.02 & .000 & 9.89 & 7.24 & .37 \\
Búsqueda de actividades & 4.23 & .000 & 8.07 & 5.16 & .38 \\
Descarga emocional & 5.47 & .000 & 7.96 & 3.37 & .47 \\
\hline
\end{tabular}

Nota. $g /(26)$. Momicidas = Media de condenados por homicidio o tentativa de homicidio; $M_{\mathrm{PG}}=$ Valor de prueba o contraste tomado de la Media de la Población General Normativa. 
presenta la totalidad de condenados por homicidio o tentativa de homicidio del C. P. de Oporto que dieron su consentimiento para participar en la investigación. Segundo, se ha considerado la muestra de condenados como un grupo homogéneo, sin establecer distinciones en función de otras características sociodemográficas o delictivas que podrían resultar relevantes (e.g., homicidio doloso o negligente, reincidencia, relación con la víctima, etc.). No obstante, su reducido número imposibilitó la creación de grupos más homogéneos con base en variables que podrían mediar en la sintomatología clínica y el afrontamiento. Tercero, se ha limitado la evaluación del ajuste psicológico y de las destrezas para la resolución de problemas a un único instrumento, el SCL-90-R y el CRI-A, respectivamente. Por ende, el empleo de otras pruebas podría derivar en un registro distinto del obtenido. De facto, no se han escudriñado variables como los trastornos relacionados con sustancias (consumo de alcohol u otras drogas) ni los trastornos de la personalidad que, frecuentemente, se registran entre la población reclusa (Guillén, Roth, Alfaro y Fernández, 2015; Vicens et al., 2011). Cuarto, se desconoce si la sintomatología clínica detectada en la evaluación ya estaba presente en el momento de la comisión del crimen, o si su origen fue posterior a la detención y condena. De igual modo acontece con la capacidad de afrontamiento, esto es, se desconoce si el contexto penitenciario pudo haber ejercido algún tipo de influencia sobre las destrezas para resolver problemas. Por último y quinto lugar, se ha asumido que los participantes han cumplimentado las pruebas de manera honesta, sin que se haya ejercido ningún control sobre una potencial simulación o disimulación. En consecuencia, resulta imposible certificar la franqueza en las respuestas dadas.

Considerando estas limitaciones, se ha observado, en primer lugar, que la mayoría de los acusados por homicidio o tentativa de homicidio guardaban algún tipo de relación previa con la víctima. Estos resultados son congruentes con la literatura criminológica, la cual refiere como habitual el mantenimiento de una relación entre la víctima y el agresor (McQuade, 2014). Por otro lado, la elevada tasa de reincidencia en diferentes tipos delictivos que se halló en el estudio (superior al 40\%), revela que la muestra de condenados se caracteriza por versatilidad delictiva y tendencia a la reincidencia.
En segundo lugar, en el estudio del ajuste psicológico se ha observado que los condenados por homicidio o tentativa de homicidio mostraban puntuaciones significativamente superiores en todas las dimensiones sintomáticas del SCL-90-R, excepto en Hostilidad y Ansiedad Fóbica. Por tanto, en contraste con la población general normativa, padecen más sintomatología somática, obsesivo-compulsiva, de sensibilidad interpersonal, depresiva, ansiosa, paranoide, y psicótica. Además, en el estudio de casos se verificó una prevalencia significativamente superior de casos clínicos en la muestra homicida en todas las dimensiones, menos en la de Hostilidad. De este modo, los condenados presentan una probabilidad significativamente superior de convertirse en caso clínico. Por su parte, los resultados hallados en los índices globales mantienen la misma dirección. La muestra de condenados presenta mayor malestar y sufrimiento psíquico y psicosomático global (GSI), más amplitud y diversidad sintomática (PST), y una intensidad sintomática media superior (PSDI).

En consecuencia, los resultados extraídos de la evaluación clínica son congruentes con aquella literatura en la que se detecta una presencia elevada de sintomatología clínica entre la población reclusa (Fazel y Seewald, 2012; Vicens et al., 2011). No obstante, es altamente complejo establecer el papel que juega este mayor padecimiento sintomático en los participantes de la muestra. Conforme a lo referido en las limitaciones, se desconoce si la sintomatología registrada ya estaba presente durante la comisión del delito, o si apareció o agravó posteriormente, durante el proceso judicial o el internamiento en el centro penitenciario. Por un lado, el ingreso en la prisión y la adaptación al régimen penitenciario tienden a generar en los internos un fuerte estrés con potencial para afectarlos psicoemocionalmente (Ruiz-Hernández et al., 2015; Vilariño y Edmundo, 2013). Así, la sintomatología depresiva y la ansiosa se registran, comúnmente, en la población reclusa, y su origen se atribuye al proceso de prisionización (Botelho y Gonçalves, 2016), aunque los eventos o contextos estresantes pueden contribuir a la aparición de otras múltiples tipologías de síntomas (somáticos, psicóticos, paranoides, etc.) (APA, 2014; Fariña, Arce, Vilariño y Novo, 2014). En suma, el proceso judicial y el internamiento en el medio penitenciario pu- 
dieron haber contribuido, al menos en parte, a la aparición de la sintomatología detectada.

Otra posibilidad interpretativa de la psicopatología detectada, consiste en considerarla como un factor de riesgo del comportamiento antisocial y delictivo (Folino, 2015). En esta dirección, algunos trabajos han detectado un mayor riesgo de homicidio en personas con diagnósticos de enfermedad mental (Schanda et al., 2004; Woodward, et al., 2000), especialmente de tipo psicótico (Richard-Devantoy et al., 2009). De facto, la literatura parece revelarse congruente ante el hecho de que la combinación de ambas variables (delincuencia y enfermedad mental) contribuye a la comisión de delitos graves y violentos (Silver et al., 2008; Vilariño, Alves y Amado, 2016). En esta línea, la muestra de condenados presentaba puntuaciones significativas y una probabilidad superior de tonarse casos clínicos en dos dimensiones que recogen síntomas asociados con la esquizofrenia y los trastornos psicóticos: ideación paranoide y psicoticismo. Esto no implica que la sintomatología hallada posea por sí misma entidad suficiente como para hablar de la presencia de algún trastorno mental de orden psicótico, tan sólo que, en contraste con la población general normativa, los condenados por homicidio presentan más sintomatología propia de estos trastornos, y que de haber estado presente durante la comisión de los mismos pudo haber contribuido como factor de riesgo, al menos parcialmente, a la emisión del comportamiento homicida. Asimismo, tampoco se puede sostener que tuviese entidad suficiente como para anular las capacidades cognoscitivas y volitivas hasta el punto de substanciar una sentencia de inimputabilidad (Osuna, López-Martínez, Arce y Vázquez, 2015).

Por tanto, del estudio del ajuste psicológico se puede concluir con garantías que, en el momento de la evaluación, los condenados por homicidio o tentativa de homicidio mostraban una probabilidad superior de ser casos clínicos en la mayoría de las dimensiones sintomáticas del SCL-90-R, así como puntuaciones significativamente superiores en casi todas ellas y en los tres índices globales. Aunque no se puede establecer de manera fehaciente, por las propias características del diseño, si la sintomatología registrada contribuyó como factor de riesgo a la emisión del comportamiento homicida, si es consecuen- cia del propio proceso judicial y del cumplimiento de la condena, o que, en realidad, no guarde relación con ninguna de ambas interpretaciones. En cualquier caso, los datos revelan la necesidad de prestar ayuda a escala de salud mental para tratar la sintomatología detectada.

Del estudio de las destrezas para resolver problemas se extrae que los condenados por homicidio o tentativa de homicidio, se caracterizan por presentar un afrontamiento por evitación. Esta modalidad de afrontamiento tiende a centrarse en la emoción en lugar de en el problema. Así, se materializa en tentativas cognitivas y conductuales para evitar pensar en el estresor y sus implicaciones, o manejar los efectos asociados (Moos, 1993). En este sentido, en los resultados obtenidos se pone de manifiesto que en las cuatro estrategias de afrontamiento consideradas evitativas (evitación cognitiva, aceptación o resignación, búsqueda de actividades alternativas, y descarga emocional), los condenados presentan puntuaciones significativamente superiores. En consecuencia, en contraste con la población general normativa recurren en mayor medida a evitar pensar realistamente sobre el problema; a aceptarlo y resignarse ante el mismo; a llevar a cabo actividades o nuevas formas de satisfacción; y a la reducción de la tensión mediante la expresión de sentimientos negativos. Se trata de estrategias que no modifican el estado del problema y, por tanto, poco productivas cuando el problema al que se hace frente resulta potencialmente modificable y susceptible de ser resuelto exitosamente.

Adicionalmente, entre las cuatro estrategias de afrontamiento por aproximación (Análisis lógico, reevaluación positiva, búsqueda de orientación y apoyo, y resolución de problemas) tan sólo se han detectado diferencias en Análisis lógico, pero en dirección contraria a la anterior. Esto es, los condenados por homicidio o tentativa de homicidio obtienen puntuaciones inferiores en esta estrategia, de tal forma que desarrollan menos esfuerzos cognitivos para entender y prepararse para un estresor y sus consecuencias. Por tanto, recurren en menor medida a una estrategia útil que contribuye a abordar los problemas de forma eficaz. La capacidad para abordar un problema, analizándolo e interpretándolo, resulta crucial para frenar comportamientos impulsivos, y ajustarlos a la legalidad y convención social. Una vez más, se 
refuerza la evidencia de que tienden a acudir a un estilo de afrontamiento no dirigido a la solución real del problema.

Tal y como se había referido previamente, de la literatura se extrae que la capacidad para resolver eventos estresantes de modo eficaz constituye un factor protector de comportamiento antisocial (Andrews y Bonta, 2010). Consecuentemente, las estrategias de afrontamiento improductivo contribuyen a la aparición del comportamiento delictivo (Llorca-Mestre, Malonda-Vidal y Samper-García, 2017). En este sentido, es posible que el estilo de afrontamiento hallado en la muestra homicida haya contribuido a la explicación del comportamiento criminal. El afrontamiento de tipo evitativo, que fue el identificado en los condenados, tiende a considerarse como una forma desadaptativa o improductiva, al menos fuera del medio penitenciario (Arce et al., 2014). Aunque en algunos trabajos se sugiere que en el contexto de prisión las estrategias evitativas podrían ser efectivas (Beijersbergen et al., 2014; Van Harreveld et al., 2007), recurrir en menor medida a la estrategia de análisis lógico no parece reportar ningún aspecto positivo. Se trata de una estrategia útil dentro y fuera del medio penitenciario. Detenerse a analizar los problemas es determinante para su resolución eficaz.

En suma, los resultados del presente trabajo ponen de manifiesto una presencia mayor de sintomatología psicopatológica entre los condenados por homicidio o tentativa de homicidio en relación con la población general normativa. Aunque no se ha podido establecer fehacientemente el papel que representan estos síntomas, se han sugerido algunas posibilidades interpretativas con base en la literatura existe. En cualquier caso y con independencia de sus posibles causas o efectos, conviene establecer intervenciones terapéuticas dirigidas a optimar el estado psíquico de los reclusos. De este modo, deberían ofrecerse servicios e intervenciones adecuadas de salud mental en los centros penitenciarios. Por otro lado, la muestra de condenados se caracteriza por acudir a estrategias de afrontamiento evitativas, que la literatura tiende a calificar de desadaptativas y que ha identificado como un factor de riesgo del comportamiento antisocial (Feelgood, Cortoni y Thompson, 2005). En consecuencia, deberían implementarse programas de intervención multi- modales dirigidos a mitigar el uso de estrategias evitativas y fomentar las aproximtaivas (Beelmann y Lösel, 2006), con el fin de favorecer la reinserción social del recluso. Adicionalmente, para conseguir los mejores resultados posibles, la intervención debería ser multinivel (Arce, Seijo, Fariña y Mohamed-Mohand, 2010; Fariña, Arce y Novo, 2008).

Finalmente, con la mente puesta en investigaciones futuras que superen las limitaciones de este trabajo, convendría ampliar la medida del ajuste psicológico y de la destreza para resolver problemas con otros instrumentos que complementen, precisen o mejoren la información reportada por el SCL-90-R y el CRI-A. Adicionalmente, sería pertinente extender el estudio, con la evaluación de otros factores de riesgo que, comúnmente, se asocian con el comportamiento criminal, y de otras variables psicológicas relevantes dentro del medio penitenciario. Todo ello, con el objetivo de extraer información que permita optimizar las medidas preventivas y reeducativas del comportamiento delictivo, así como incrementar el bienestar del interno.

\section{Referencias}

American Psychiatric Association. (2002). Manual diagnóstico y estadístico de los trastornos mentales [Diagnostic and Statistical Manual of Mental Disorders] (4a Ed., texto rev.). Barcelona, España: Masson.

American Psychiatric Association. (2014). Manual diagnóstico y estadístico de los trastornos mentales $(D S M-V)$ [Diagnostic and Statistical Manual of Mental Disorders (DSM-V]). Madrid, España: Panamericana.

Andrews, D. A. y Bonta, J. (2010). The psychology of Criminal Conduct (5a. Ed.). Cincinnati, OH: Anderson Publishing Co.

Arce, R., Fariña, F. y Novo, M. (2014). Cognitive Competence among Recidivist and Non-Recidivist Prisoners: Implications for the Rehabilitation. 
Anales de Psicología, 30, 259-266. https://doi.org/10.6018/analesps.30.1.158201

Arce, R., Seijo, D., Fariña, F. y Mohamed-Mohand, L. (2010). Comportamiento antisocial en menores: Riesgo social y trayectoria natural de desarrollo [Antisocial Behaviour in Adolescents: Social Risk and Natural Development Trajectory]. Revista Mexicana de Psicología, 27, 127-142.

Arce, R., Fariña, F., Seijo, D., Novo, M. y Vázquez, M. J. (2005). Contrastando los factores de riesgo y protectores del comportamiento inadaptado en menores: Implicaciones para la prevención [Contrasting Risk Factors and Protectors of Inappropriate Behaviour in Minors: Implications for Prevention]. En Centro de Investigación y Documentación Educativa (Ed.), Premios Nacionales de Investigación Educativa 2004 (pp. 17-50). Madrid, España: Ministerio de Educación y Ciencia.

Arce, R., Fariña, F. y Vázquez, M. J. (2011). Grado de competencia social y comportamientos antisociales delictivos y no delictivos en menores [Social Competence and Delinquent, Antisocial, and NonDeviant Behavior in Adolescents]. Revista Latinoamericana de Psicología, 43(3), 473-486. Recuperado de http://www.scielo.org.co/pdf/rlps/v43n3/v43n3a07. pdf

Beelmann, A. y Lösel, F. (2006). Child Social Skills Training in Developmental Crime Prevention: Effects on Antisocial Behavior and Social Competence. Psicothema, 18, 603-610.

Beijersbergen, K. A., Dirkzwager, A. J. E., Eichelsheim, V. I., Van der Laan, P. H. y Nieuwbeerta, P. (2014). Procedural Justice and Prisoners' Mental Health Problems: A Longitudinal Study. Criminal Behaviour and Mental Health, 24, 100-112. https://doi.org/10.1002/cbm.1881

Botelho, M. y Gonçalves, R. A. (2016). Why do People kill? A Critical Review of the Literature on Factors
Associated with Homicide. Aggression and Violent Behavior, 26, 9-15. https://doi.org/10.1016/j.avb.2015.11.001

Cohen, J. (1988). Statistical power Analysis for the Behavioral Sciences (2a ed.). Hillsdale, NJ: LEA.

Connell, C. M., Cook, E. C., Aklin, W. M., Vanderploeg, J. J. y Brex, R.A. (2011). Risk and Protective Factors Associated with Patterns of Antisocial Behavior among Nonmetropolitan Adolescents. Aggressive Behavior, 37, 98-106. https://doi.org/10.1002/ab.20370

Derogatis, L.R. (1983). SCL-90-R: Administration, scoring, and Procedures Manual II. Baltimore: Clinical Psychometric Research.

Eronen, M., Hakola, P. y Tiihonen, J. (1996). Mental Disorders and Homicidal Behavior in Finland. Archives of General Psychiatry, 53, 497-501. https://doi.org/10.1001/archpsyc.1996.01830060039 005

Eurostat Statistics Database. (2014, enero). Estadísticas sobre la delincuencia. Recuperado de http://ec.europa.eu/eurostat/statisticsexplained/index.php/Crime_statistics/es

Fariña, F., Arce, R. y Novo, M. (2008). Neighborhood and Community Factors: Effects on Deviant Behavior and Social Competence. The Spanish Journal of Psychology, 11, 78-84. https://doi.org/10.1017/S1138741600004133

Fariña, F., Arce, R., Vilariño, M. y Novo, M. (2014). Assessment of the Standard Forensic Procedure for the Evaluation of Psychological Injury in IntimatePartner Violence. Spanish Journal of Psychology, 17 (e32), 1-10. https://doi.org/10.1017/sjp.2014.30

Farrington, D. P. y Ttofi, M. M. (2012). Protective and Promotive Factors in the Development of Offending. En T. Bliesener, A. Beelmanny M. Stemmler (Eds.), Antisocial Behavior and Crime: Contributions of Developmental and Evaluation 
Research to Prevention and Intervention (pp. 7188). Cambrigde, MA: Hogrefe Publishing.

Fazel, S. y Grann, M. (2004). Psychiatric Morbidity among Homicide Offenders: A Swedish Population Study. American Journal Psychiatry, 161, 21292131. https://doi.org/10.1176/appi.ajp.161.11.2129

Fazel, S. y Seewald, K. (2012). Severe Mental Illness in 33588 Prisoners Worldwide: Systematic Review and Meta-Regression Analysis. British Journal of Psychiatry, 200, 364-373. https://doi.org/10.1192/bjp.bp.111.096370

Feelgood, S., Cortoni, F. y Thompson, A. (2005). Sexual Coping, General Coping, and Cognitive Distortions in Incarcerated Rapist and Child Molesters. Journal of Sexual Aggression, 11, 157-170. https://doi.org/10.1080/13552600500073657

Folino, J. O. (2015). Predictive Efficacy of Violence Risk Assessment Instruments in Latin-America. European Journal of Psychology Applied to Legal Context, $7, \quad 51-58$. https://doi.org/10.1016/j.ejpal.2014.11.006

Glied, S. y Frank, R. G. (2014). Mental Illness and Violence: Lessons from the Evidence. American Journal of Public Health, 104, e5-e6. https://doi.org/10.2105/AJPH.2013.301710

Guillén, N., Roth, E., Alfaro, A. y Fernández, E. (2015). Youth Alcohol Drinking Behavior: Associated Risk and Protective Factors. Revista Iberoamericana de Psicología y Salud, 6(2), 53-63. https://doi.org/10.1016/j.rips.2015.03.001

Hedges, L. V. y Olkin, I. (1985). Statistical Methods for Meta-Analysis. San Diego, CA: Academic Press.

Kirchner, T., Forns, M. y Mohíno, S. (2007). Psychological Adjustment in a Forensic Sample: Relationship with Approach- and AvoidanceCoping Typologies. Journal of Forensic Science, 52, 712-716. https://doi.org/0.1111/j.15564029.2007.00430.x
Llorca-Mestre, A., Malonda-Vidal, E. y Samper-García, P. (2017). Prosocial Reasoning and Emotions in Young Offenders and Non-Offenders. European Journal of Psychology Applied to Legal Context, 9(2), 65-73. https://doi.org/10.1016/j.ejpal.2017.01.001

McMurran, M. y Christopher, G. (2009). Social Problem Solving, Anxiety, and Depression in Adult Male prisoners. Legal and Criminological Psychology, 14 , 101-107. https://doi.org/10.1348/135532507X267031

McQuade, K. M. (2014). Victim-Offender Relationship. The Encyclopedia of Criminology and Criminal Justice (pp. $1-5)$. https://doi.org/10.1002/9781118517383.wbeccj131

Moore, T. M., Eisler R. y Franchina, J. J. (2000). Causal Attributions and Affective Responses to Provocative Female Partner Behavior by Abusive and Nonabusive Males. Journal of Family Violence, 15 , 69-80. https://doi.org/10.1023/A:1007501520734

Moos, R. (1993). Coping Response Inventory Adult Form-Professional. Manual. Florida: Psychological Assessment Resources.

Osuna, E., López-Martínez, M., Arce, R. y Vázquez, M. J. (2015). Analysis of Response Patterns on the MMPI-2 in Psychiatric Prison Inmates. International Journal of Clinical and Health Psychology, 15, 29-36. https://doi.org/10.1016/j.ijchp.2014.09.002

Richard-Devantoy, S., Olie, J. P. y Gourevitch, R. (2009). Risk of Homicide and Major Mental Disorders: A Critical Review. Encephale, 35, 521530. https://doi.org/10.1016/j.encep.2008.10.009

Rosenthal, R. (1994). Parametric Measures of Effect Size. En H. Cooper y L. V. Hedges (Eds.), The handbook of research synthesis (pp. 231-244). New York, NY: Russell Sage Foundation. 
Ruiz-Hernández, J. A., García-Jiménez, J. J., LlorEsteban, B. y Godoy-Fernández, C. (2015). Factores de riesgo de violencia de pareja en población penitenciaria [Risk Factors for Intimate Partner Violence in Prison Inmates]. European Journal of Psychology Applied to Legal Context, 7, 41-49. https://doi.org/10.1016/j.ejpal.2014.11.003

Schanda, H., Knecht, G., Schreinzer, D. Stompe, T., Ortwein-Swoboda, G. y Waldhoer, T. (2004). Homicide and Major Mental Disorders: A 25-year Study. Acta Psychiatrica Scandinavica, 110, 98$107 . \quad$ https://doi.org/10.1111/j.16000047.2004.00305.x

Silver, E., Felson, R. B. y Vaneseltine, M. (2008). The Relationship between Mental Health Problems and Violence Among Criminal Offenders. Criminal Justice and Behavior, 35, 405-426. https://doi.org/10.1177/0093854807312851

Van Harreveld, F., Van der Pligt, J., Claassen, L. y Van Dijk, W. W. (2007). Inmate Emotion Coping and Psychological and Physical Well-Being. The use of Crying Over Spilled Milk. Criminal Justice and Behavior, 34, 697-708. https://doi.org/10.1177/0093854806298468

Vicens, E., Tort, V., Dueñas, R. M., Muro, A., PérezArnau, F., Arroyo, J. M., ...Sarda, P. (2011). The
Prevalence of Mental Disorders in Spanish Prisons. Criminal Behaviour and Mental Health, 21, 321332. https://doi.org/10.1002/cbm.815

Vilariño, M., Alves, A. C. y Amado, B. G. (2016). Perfil clínico y delictivo de una muestra de reclusos inimputables de Portugal [Clinical and Criminal Profile in a Sample of Legal Insanity Portugues Inmatesl]. En A. Andrés-Pueyo, F. Fariña, M. Novo y D. Seijo (Eds.), Avances en psicología jurídica y forense (pp. 111-121). Santiago de Compostela, España: Sociedad Española de Psicología Jurídica y Forense.

Vilariño, M. y Edmundo, M. (2013). Estudio de la salud mental en una muestra de reclusos portugueses. En F. Expósito, I. Valor-Segura, M. Vilariño y A. Palmer (Eds.), Psicología jurídica aplicada a los problemas sociales (pp. 217-222). Santiago de Compostela, España: Sociedad Española de Psicología Jurídica y Forense.

Woodward, M., Nursten, J., Williamns, P. y Badger, D., (2000). Mental Disorder and Homicide: A Review of Epidemiological Research. Epidemiology and Psychiatric Sciences, 9, 171-189. https://doi.org/10.1017/S1121189X00007867 


\title{
PSYCHOLOGICAL ADJUSTMENT AND PROBLEM-SOLVING SKILLS IN CONVICTED BY MURDER OR ATTEMPTED MURDER
}

\author{
MANUEl VilARiÑO ${ }^{1}$, BARBARA G. AMADO ${ }^{2}$, ElsA FERNANDES ${ }^{2}$ \\ Y RAMÓN ARCE ${ }^{3}$
}

\section{EXTENDED SUMMARY}

\section{Introduction}

Mental illness is one of the risk factors associated to criminal behaviour (Arce, Fariña, \& Novo, 2014). The relationship between both variables was extendedly investigated with contradictory results. Thus, high prevalence rates of mental disorders were found in prison inmates (Fazel \& Grann, 2004; Fazel \& Seewald, 2012), as well as a higher risk of homicide behaviours in individuals with a mental disorder diagnosis (Eronen, Hakola, \& Tiihonen, 1996; Schanda et al., 2004; Woodward, Nursten, Williamns, \& Badger, 2000), mainly a psychotic disorder, antisocial disorder and alcohol or drug abuse (Richard-Devantoy, Olie, \& Gourevitch, 2009; Woodward, et al., 2000). Nonetheless, literature is more consistent in determining the facilitator role of the combination of mental illness and delinquency variables in developing violent behaviours in contrast when they appear isolated (Vicens et al., 2011).

Notwithstanding, prison inmate mental illness high prevalence rates can be either cause or effect of imprisonment. The impact of prisonization on inmate's health generally causes sensory and personal image disturbances, loss of self-esteem, depersonalisation, sexual disturbances and anxious and depressive symptomatology (Botelho \& Gonçalves, 2016; Ruiz-Hernández, GarcíaJiménez, Llor-Esteban, \& Godoy-Fernández, 2015; Vilariño \& Edmundo, 2013).
Problem-solving coping strategies represent a risk factor for criminal behaviour (Arce et al., 2014; Moore, Eisler, \& Franchina, 2000). Overall, investigation has pointed out that antisocial behaviour is closely related to dysfunctional and inefficient coping strategies, in both in juvenile offenders and minors at risk of delinquency (Arce, Fariña, Seijo, Novo, \& Vázquez, 2005; Vilariño, Amado, \& Alves, 2013), as well as in prison inmates (Arce et al., 2014).

On the other hand, assuming adaptive coping strategies in prison could play a relevant role in prison inmates' physical and mental health. Likewise, the relationship between coping and psychological well-being or mental health in prison inmates have been investigated in depth. Contradictory results have been reached again, without a consensus regarding the way coping strategies affect inmates' psychological well-being (McMurran \& Christopher, 2009; Kirchner, Forns, \& Mohíno, 2007).

The aim of the present study was to progress on risk factors knowledge which underlies criminal behaviour, namely, homicide or attempted homicide. Furthermore, it is expected to obtain useful knowledge to create preventing intervention, re-education or therapeutic programs. Therefore, a study to contrast psychological adjustment (clinical symptomatology), and problem-solving skills (coping strategies) in a male sample of convicted by murder or attempted murder with the normative sample was carried out. Additionally, with the objective of extending more information about the prison inmates, archive data related to the type of homicide committed, the relationship with the victim and relapse were gathered. 


\section{Method}

\section{Participants}

Participants were 27 male prison inmates, ranging age from 22 to 65 years $(M=38.63 ; S D=10.72)$, convicted by homicide or attempted homicide All they were serving the sentence at the 'Estabelecimento Prisional do Porto' (Prison of Oporto, Portugal).

\section{Design and Procedure}

A study focusing on clinical symptomatology measured in a sentenced homicide or attempted homicide sample was designed. The procedure consisted on contrast the prison inmates' mental health state with the SCL-90-R normative population (Derogatis, 2002). Likewise, coping skills were measured through CRI-A questionnaire in the same sample of participants, which was compared again with the normative population (Moos, 1993).

The sentenced sample was accidentally selected in the Prison of Oporto, and their participation was voluntary and authorized. Thus, the sample was made up of the whole sentenced murderers or attempted murders that came from that prison, and they gave informed consent to participate in the evaluation.

\section{Instrumental measures}

The Portuguese translation (back translation procedure) of the SCL-90-R [Symptom Check List-90-R] from Derogatis (2002) to evaluate clinical symptomatology and the CRI-A [Coping Responses Inventory Adult Form] from Moos (1993) to measure coping strategies, were the psychometric tests applied in the present study. The SCL-90-R was developed to assess symptoms patterns present on the individual.

\section{Data Analysis}

To contrast the murderers' scores (clinical symptomatology, and coping skills) with normative general population scores (test value), $t$ test was used. Thus, the study sample was compared with a given test value, and $t$ test was transformed on $r$ effect size (Cohen, 1988; Rosenthal, 1994). With the aim to contrast the observed proportion with a test value (registered clinical cases in general population), $Z$ tests were calculated and thereafter $\delta$ effect size (Hedges \& Olkin, 1985). Additionally, symptomatology's increment from one group respect to another (prison inmates vs. normative population), was obtained directly from $r$ effect size.

\section{Results}

\section{Criminological characteristics of mur- derers or attempted murderers}

The prison inmates' criminal records were used to extract the following data. From 27 overall participants, $55.5 \%$ (13) had committed a homicide, $44.4 \%$ (12) intentional homicide, and $11.1 \%$ (3) involuntary manslaughter. . The remaining $44.4 \%$ was sentenced for attempted homicide. Regarding the relationship with the victim, 59.3\% (16) had a previous relationship (11.1\% partner, $11.1 \%$ family, $37 \%$ friendship/neighbourhood relationship/fellowship). Additionally, $55.6 \%$ of prison inmates lack of criminal records, and from those who had criminal records only one $(3.7 \%)$ had relapsed in the same offence.

\section{Clinical symptomatology contrast in murderers or attempted murderers im- prisoned and normative population in $S C L-90-R$}

Regarding the SCL-90-R symptomatic dimensions, significant results were obtaining between scores from the prison inmates sample and the SCL-90-R normative 
population in all, exception of Hostility and Phobic Anxiety dimensions (see Table 2). Such differences were significantly higher in the convicted sample in Somatization, Obsessive-Compulsive, Interpersonal Sensitivity, Depression, Anxiety, Paranoid Ideation and Psychoticism dimensions.

Moreover, significant differences have arisen in the SCL-90-R global indexes: GSI, PST and PSDI (see Table $3)$. In line with the symptomatic dimension results, the sentenced murderer or attempted murder sample had informed of a higher symptomatic suffering.

\section{Clinical cases study and SCL-90-R symptomatic dimensions}

Results in the SCL-90-R symptomatic dimensions have shown a higher significant prevalence of clinical cases, in all with the exception of Hostility, in the sentenced murderers or attempted murderers' sample (see Table 4).

\section{Coping skill contrast between convic- ted murderers or attempted murder and normative population on CRI-A}

Contrast in coping skills (see Table 5) has exhibited differences in 6 out of 8 CRI-A scales. Normative population only displayed one higher significant score in the Logical Analysis scale. In the remaining scales with significant differences, that is, Cognitive Avoidance, Acceptance or Resignation, Seeking Alternative Rewards and Emotional Discharge, the sentenced sample presented higher significant scores.

\section{Discussion}

It was verified, from the study of criminal characteristics, that most of the prison inmates have had a previous relationship with the victim. Furthermore, they were characterized by a criminal versatility and recidivism tendency.

Likewise, from the psychological adjustment analysis (clinical symptomatology) we can conclude with guarantees that, at the time of the evaluation, sentenced murderers or attempted murderers shown a higher probability of being classified as clinical cases in most of the SCL-90-R symptomatic dimensions, as well as a higher probability of informing about higher significant scores in almost every scale and in all global indexes. However, owing to the design characteristics, it could not be established irrefutably whether the informed symptomatology was as a risk factor facilitating the homicide behaviour, whether it is a consequence of the judicial processes and the criminal sentence compliance, or even it is not related to none of both interpretations.

Finally, solving-problem skill study conclusions (coping strategies) have shown that sentenced murderers or attempted murderers' sample was characterized by using an avoidance coping strategy. This coping style tends to focus on emotion instead of on problem. Thus, it is reflected on cognitive and behavioural attempts to avoid thinking in the stressor and its implications, or to manage associated effects (Moos, 1993). In this sense, results demonstrated that in all four avoidance coping strategies (cognitive avoidance, acceptance or resignation, seeking alternative rewards and emotional discharge), sentenced prison inmates reported higher significant scores. These strategies do not modify the state of the problem, becoming unproductive when such coping problem turns out modifiable and susceptible to be successfully solved. Additionally, Logical Analysis was the only approximation coping strategy that have shown differences but in the contrary direction. Sentenced murderers or attempted murderers scored less in such strategy, that is, they make less cognitive efforts to understand and face the stressor and its consequences. Therefore, they turn to a lesser extent, useful strategy that contributes to solve problems efficiently. Once again, evidence reinforced that prison inmates have a coping style that not address the real trouble. According to previous researches, maladaptive coping strategies contribute at the emergence of criminal behaviour (Andrews \& Bonta, 2010; Llorca-Mestre, Malonda-Vidal, \& Samper-García, 2017). Avoidance 
coping style, which was characteristic of the sentenced sample, is considered as a maladaptive or unproductive strategy, at least out of prison context (Arce et al., 2014). In this sense, it is probable that avoidance coping style has contributed to the criminal behaviour explanation in the sentenced sample.

To summarize, results of the present study exhibit a wide range of psychopathological symptomatology in sentenced murderers or attempted murderers in contrast to the normative population. Thus, adequate mental health interventions and services in prison should be offered. On the other hand, convicted sample is characterized by using avoidance coping strategies. As a consequence and with the objective of facilitating prison inmates' social reintegration, multimodal intervention programs driven to mitigate avoidance coping strategies and to empower more adaptive coping strategies i.e., approximate ones, should be implemented (Beelmann \& Lösel, 2006),. Additionally, multi-level intervention must be implemented in order to reach the best possible outcomes (Arce, Seijo, Fariña, \& Mohamed-Mohand, 2010; Fariña, Arce, \& Novo, 2008). 